\title{
Editorial: Understanding the Importance of Non-Canonical tRNA Function
}

\author{
Juan Pablo Tosar ${ }^{1,2 * t}$, Pavel Ivanov ${ }^{3,4,5 * t}$, Lluís Ribas de Pouplana ${ }^{6,7 * t}$ and \\ Adrian Gabriel Torres ${ }^{7 * t}$
}

${ }^{1}$ Functional Genomics Unit, Institute Pasteur de Montevideo, Montevideo, Uruguay, ${ }^{2}$ Faculty of Science, Universidad de la República, Montevideo, Uruguay, ${ }^{3}$ Division of Rheumatology, Immunology and Allergy, Brigham and Women's Hospital, Boston, MA, United States, ${ }^{4}$ Department of Medicine, Harvard Medical School, Boston, MA, United States, ${ }^{5}$ The Broad Institute of Harvard and M.I.T., Cambridge, MA, United States, ${ }^{6}$ Catalan Institution for Research and Advanced Studies, Barcelona, Spain, ${ }^{7}$ Institute for Research in Biomedicine, The Barcelona Institute of Science and Technology, Barcelona, Spain

Keywords: transfer RNA, tRNA-derived fragments, tRNA modifications, tRNA processing, non-canonical function, gene regulation, human diseases, evolution

\section{OPEN ACCESS}

Edited and reviewed by: André P. Gerber,

University of Surrey, United Kingdom

*Correspondence: Juan Pablo Tosar jptosar@pasteur.edu.uy Pavel Ivanov pivanov@rics.bwh.harvard.edu

Lluís Ribas de Pouplana

Iluis.ribas@irbbarcelona.org

Adrian Gabriel Torres

adriangabriel.torres@ irbbarcelona.org

${ }^{\dagger}$ These authors have contributed equally to this work

Specialty section:

This article was submitted to Protein and RNA Networks,

a section of the journal

Frontiers in Molecular Biosciences

Received: 02 September 2021 Accepted: 21 September 2021 Published: 07 October 2021

Citation:

Tosar JP, Ivanov $P$ Ribas de Pouplana $L$ and Torres AG (2021) Editorial: Understanding the Importance of Non-Canonical tRNA Function.

Front. Mol. Biosci. 8:769784. doi: 10.3389/fmolb.2021.769784

\section{Editorial on the Research Topic}

\section{Understanding the Importance of Non-Canonical tRNA Function}

The last universal common ancestor of all current forms of life already contained transfer RNAs (tRNAs) serving as adaptor molecules between nucleic acids and proteins. However, only in recent years has it become clear that the functions of these ancient RNA molecules expand beyond their central role in protein synthesis by the ribosome (Schimmel, 2018). The goal of this Research Topic is to revise our current knowledge on these non-canonical tRNA functions and create a forum for discussion that would expand and stimulate this field of research. Here, we are delighted to present a Research Topic of articles that address the importance of non-canonical tRNA functions from molecular, biochemical, evolutionary, and biomedical perspectives.

In the review by Avcilar-Kucukgoze and Kashina, key non-canonical tRNA functions in eukaryotes are introduced and elegantly presented. The focus is placed on the involvement of tRNAs in diverse cellular processes such as stress response triggered by amino acid starvation, regulation of mitochondria-triggered apoptosis, protein arginylation and other tRNAmediated post-translational modifications, priming of retrotransposons, adaptive mistranslation by tRNA mischarging, and regulation of biological processes by tRNAderived fragments (tRFs) including tRNA-derived stress-induced RNAs (tiRNAs) and tRNA halves (Ivanov et al., 2011).

Extracellular RNAs (ex-RNAs) are involved in cell-to-cell communication and may act as a potential source of biomarkers for human diseases, but the roles of ex-tRNAs or their fragments (extRFs) in these processes have not been widely studied, despite being among the most abundant exRNAs (Tosar et al., 2020). Torres and Martí discuss the biology of extracellular tRNAs and tRFs and propose a critical role for post-transcriptional tRNA modifications in ex-tRNA/ex-tRF recognition, stability, uptake and function in recipient cells.

MicroRNAs (miRNAs) are small non-coding RNAs that associate with argonaute (Ago) proteins and repress translation upon binding to target messenger RNAs (mRNAs). MiRNA/mRNA interactions are based on binding of the $5^{\prime}$-end of the miRNA to the $3^{\prime}$-end region of mRNAs (herein, $5^{\prime}$ miRNA-mRNA forward orientation). Previous reports have shown that certain tRFs can perform miRNA-like Ago-mediated mRNA silencing (Kumar et al., 2014; Kuscu et al., 2018; Guan et al., 2020). However, tRF/mRNA recognition rules are still not completely understood. Guan et al. 
analyzed CLASH (crosslinking, ligation, and sequencing of hybrids) and Ago PAR-CLIP (photoactivatable ribonucleosideenhanced crosslinking and immunoprecipitation) datasets, in order to identify potential motifs dictating tRF/mRNA interactions. The novelty of their work rely on considering not only forward ( $5^{\prime}$ tRF-mRNA), but also reverse ( $5^{\prime}$ mRNA-tRF) oriented CLASH chimeras. By doing so, the authors improved the discovery of sequence motifs potentially involved in tRF/mRNA interaction, found a clear asymmetry of the paired tRF and their targets in both orientations suggesting alternative modes of tRF/ mRNA recognition, and provided a comprehensive list of candidate tRFs with target-binding motifs worth for future experimental validation.

Analyses of tRNA gene (tDNA) composition reveal a high redundancy at the organismal level that cannot be fully explained by the need to express cognate tRNAs (Torres, 2019) (see also below). Furthermore, some organisms (i.e., Archaea) even reconstitute their tRNAs from split tDNAs. These split genes code for different tRNA parts that can assemble into the full-length mature tRNA posttranscriptionally, and could possibly be an ancestral trait rather than a group-specific genomic innovation (Kanai, 2015). In an opinion article, Grigoriev explores the possibility that tRFs could be precursors to modern RNA interference (RNAi) mechanisms based on analyses of tDNA patterns. Transcripts deriving from tDNA-like genes and split tDNAs could have been a source for small non-coding RNAs (i.e., tRFs) that could have been loaded into primordial Ago proteins and exert early RNAi-like functions. This would support the function of tRFs being ancestral, possibly preceding the hypothetical genomic condensation event of split tRNA genes into modern tDNAs. Because the sequence of tRFs is constrained by their parental tRNA structure, new hairpins could have evolved from non-coding genomic regions later in evolution, thus providing a new potential source of small RNAs with higher sequence flexibility (i.e., extant small regulatory RNAs). Nevertheless, tRFs are still involved in fundamental processes to date, so they would have likely conserved part of their original functions.

Analyses of tDNA variants can also give further insights into potential non-canonical tRNA functions. The work by Ehrlich et al. studies tDNA composition in the three domains of life and

\section{REFERENCES}

Grosjean, H., de Crécy-Lagard, V., and Marck, C. (2010). Deciphering Synonymous Codons in the Three Domains of Life: Co-evolution with Specific tRNA Modification Enzymes. FEBS Lett. 584 (2), 252-264. doi:10.1016/j.febslet.2009.11.052

Guan, L., Karaiskos, S., and Grigoriev, A. (2020). Inferring Targeting Modes of Argonaute-Loaded tRNA Fragments. RNA Biol. 17 (8), 1070-1080. doi:10.1080/15476286.2019.1676633

Ivanov, P., Villen, J., Gygi, S. P., Anderson, P., and Anderson, P. (2011). Angiogenin-induced tRNA Fragments Inhibit Translation Initiation. Mol. Cel 43 (4), 613-623. doi:10.1016/j.molcel.2011.06.022

Kanai, A. (2015). Disrupted tRNA Genes and tRNA Fragments: A Perspective on tRNA Gene Evolution. Life 5 (1), 321-331. doi:10.3390/life5010321 focuses on those clades that are missing specific tRNA genes. Some tDNAs are lost to prevent decoding ambiguities. For example tDNAs encoding tRNAs with $A$ or $G$ at the first position of the anticodon tend to be mutually exclusive (Grosjean et al., 2010; Novoa et al., 2012). However, exceptions to such anticodon-sparing strategies exist in several species (Maraia and Arimbasseri, 2017), questioning the strength of the selection forces that shaped tDNA-loss patterns. By using updated tRNA gene databases coupled to manual curation of predicted tDNAs, the authors found that many of these exceptions were tDNAs that were unlikely to produce bona fide tRNAs. Although much of the evolutionary pressure shaping tRNA gene features is probably linked to translation fidelity (i.e., canonical tRNA function), the authors made a case that $\mathrm{tDNAs}$ that can potentially produce a priori non-bona fide tRNAs may be a source for tRNA variants with non-canonical functions.

More comprehensive and reliable methods for tRNA/tRF detection and quantification are being rapidly developed. These now also include the possibility of detecting and mapping post-transcriptional RNA modifications at singlenucleotide resolution, and evaluating tRNA processing steps. Thus, we are looking forward to new technical and conceptual developments that will surely push the field beyond its current status. Meanwhile, we invite the readers to dive into this Research Topic of papers to get a glimpse of the function of tRNAs beyond translation. Even if these functions represent just the tip of the iceberg of what is still left to be uncovered.

\section{AUTHOR CONTRIBUTIONS}

JT and AT drafted the manuscript, PI and LR modified and improved the manuscript. All authors have approved the manuscript.

\section{ACKNOWLEDGMENTS}

We thank all the authors that have contributed to this Research Topic.

Kumar, P., Anaya, J., Mudunuri, S. B., and Dutta, A. (2014). Meta-analysis of tRNA Derived RNA Fragments Reveals that They Are Evolutionarily Conserved and Associate with AGO Proteins to Recognize Specific RNA Targets. BMC Biol. 12, 78. doi:10.1186/s12915-014-0078-0

Kuscu, C., Kumar, P., Kiran, M., Su, Z., Malik, A., and Dutta, A. (2018). tRNA Fragments (tRFs) Guide Ago to Regulate Gene Expression posttranscriptionally in a Dicer-independent Manner. RNA 24 (8), 1093-1105. doi:10.1261/rna.066126.118

Maraia, R., and Arimbasseri, A. (2017). Factors that Shape Eukaryotic tRNAomes: Processing, Modification and Anticodon-Codon Use. Biomolecules 7 (1), 26. doi:10.3390/biom7010026

Novoa, E. M., Pavon-Eternod, M., Pan, T., and Ribas de Pouplana, L. (2012). A Role for tRNA Modifications in Genome Structure and Codon Usage. Cell 149 (1), 202-213. doi:10.1016/j.cell.2012.01.050 
Schimmel, P. (2018). The Emerging Complexity of the tRNA World: Mammalian tRNAs beyond Protein Synthesis. Nat. Rev. Mol. Cel Biol. 19 (1), 45-58. doi:10.1038/nrm.2017.77

Torres, A. G. (2019). Enjoy the Silence: Nearly Half of Human tRNA Genes Are Silent. Bioinform. Biol. Insights 13, 117793221986845. doi:10.1177/ 1177932219868454

Tosar, J. P., Segovia, M., Castellano, M., Gámbaro, F., Akiyama, Y., Fagúndez, P., et al. (2020). Fragmentation of Extracellular Ribosomes and tRNAs Shapes the Extracellular RNAome. Nucleic Acids Res. 48 (22), 12874-12888. doi:10.1093/nar/gkaa674

Conflict of Interest: The authors declare that the research was conducted in the absence of any commercial or financial relationships that could be construed as a potential conflict of interest.
Publisher's Note: All claims expressed in this article are solely those of the authors and do not necessarily represent those of their affiliated organizations, or those of the publisher, the editors and the reviewers. Any product that may be evaluated in this article, or claim that may be made by its manufacturer, is not guaranteed or endorsed by the publisher.

Copyright $\odot 2021$ Tosar, Ivanov, Ribas de Pouplana and Torres. This is an openaccess article distributed under the terms of the Creative Commons Attribution License (CC BY). The use, distribution or reproduction in other forums is permitted, provided the original author $(s)$ and the copyright owner(s) are credited and that the original publication in this journal is cited, in accordance with accepted academic practice. No use, distribution or reproduction is permitted which does not comply with these terms. 